\title{
Editorial: A service versus an approach: the importance of building primary palliative care
}

Nurses across all specialities are uniquely placed to positively contribute to addressing the many unmet needs that patients and their families have at the end of life. Over the past three decades there have been many positive developments as a result of the global emergence of specialist palliative care services. These services have raised the profile of best end-of-life care practices and afforded comfort and dignity to many people. However, the downside is that palliative care has in many ways become synonymous with a 'service' as opposed to being an 'approach to care' that can be provided by the patient's usual care team. As a consequence there is a perception in many countries that 'everyone who needs palliative care' ought to be cared for by a specialist palliative care team and that palliative care is reserved for people who are in the last weeks or days of life. Neither of which is correct or particularly helpful, especially for the many patients and families who would benefit from the introduction of a palliative approach to care earlier in their illness trajectory.

Unfortunately, this perception effectively limits the input of the team that probably knows the patients and their families best: that is, their usual care team. However, as specialist palliative nurses we need to be supporting and promoting the development of the patients' primary palliative care team to provide a palliative approach which will enable them to: identify and address the patients' and carers' unmet needs; manage their symptoms; minimise carer burden, clarify goals of care and align treatment, as well as determining when other specialist or palliative care input is indicated. The adoption of a palliative approach allows the primary palliative care team to maximise the quality of life for people with advanced chronic conditions and facilitate joint decision-making about future care.

Building the primary palliative care teams' palliative approach capabilities requires positive public policy that supports and rewards the delivery of a population-based approach to palliative care by specialist services. This policy should include a reorientation of specialist services so they focus their attention on patients and families with the most complex palliative care needs and are better able to support primary palliative care providers to provide a palliative approach. Building the capacity of primary palliative care teams globally is crucial to extending the reach of palliative care so that more patients and their families will have their constellation of physical, psychosocial and spiritual needs addressed in a timely manner by the team that knows them best. The integration of palliative approach learning content into undergraduate curricula is important in terms of better preparing the emerging nursing workforce to feel confident to respond to their patients' palliative care needs.

The contribution that nurses working outside of specialist settings are making to the provision of primary palliative care must be acknowledged. Gaining insights into their experiences, needs and an improved understanding of the challenges they face helps specialist palliative care nurses to be better equipped to support our colleagues in their primary palliative care roles and to build their palliative care capabilities. This in turn, allows specialist palliative care nurses to focus their attention on caring for patients and families with the most complex palliative care needs, regardless of age, diagnosis or care setting.

Jane Phillips Professor Nursing (Palliative Care), Director of the Centre for Cardiovascular and Chronic Care at University of Technology Sydney 